УДК 550.4:546.44

МОДЕЛИРОВАНИЕ ПОДВИЖНОСТИ РАДИЯ-226

ПО ДАННЫМ ЕГО ПРОФИЛЬНОГО РАСПРЕДЕЛЕНИЯ В ЗАГРЯЗНЕННОЙ ПОДЗОЛИСТОЙ ПОЧВЕ

Рачкова Н.Г., Шапошникова Л.М.

Институт биологии ФИЦ Коми НЦ УрО РАН, Сыктывкар, e-mail: nata.rachkova67@mail.ru

\begin{abstract}
С целью моделирования поведения поллютантов в загрязненных экосистемах таёжной зоны исследованы долговременное распределение и трансформация форм нахождения радия-226 в верхней 50-сантиметровой толще типичной подзолистой почвы, изучены статистические связи параметров этих процессов и физико-химических характеристик почвы. Выявлено, что при многолетнем контакте водорастворимые соединения радионуклида трансформируются и прочно связываются в почвенном поглощающем комплексе. Основной запас мобильных форм нахождения радия депонируется в слое почвы (0-20) см в составе потенциально подвижных соединений, экстрагирующихся 1 моль/дм³ соляной кислотой, что важно для выбора методов дезактивации грунтов, сходных по физико-химическому составу и типу процессов почвообразования. В горизонты почвы глубже 20 см мигрирует меньше $11,2 \%$ радия от его количества в толще (0-50) см. Суммарно в кислоторастворимой и фиксированной фракциях сосредоточено 95\% запаса радия, легкоподвижные водорастворимые и обменные формы радионуклида обеспечивают 1 и 4\% его количества. Результаты линейного регрессионного и корреляционного анализов свидетельствуют, что в условиях радиоактивного загрязнения профильная дифференциация радия в почвах подзолистого типа контролируется процессами распределения соединений кальция. Для аппроксимации показателей миграции радия предложены математические модели, качество которых оценено значениями коэффициентов детерминации от 0,467 до 0,997. Результатами моделирования показано, что взаимодействие соединений железа и кальция определяет подвижность радионуклида в загрязненной почве. Полученные данные могут быть востребованы для прогнозирования распространения и локализации радия в экосистемах.
\end{abstract}

Ключевые слова: радиоактивное загрязнение, подзолистая почва, радий-226, валовое содержание, формы нахождения, профильное распределение, регрессионный анализ

\title{
MODELING THE MOBILITY OF RADIUM-226 BASED ON THE DATA OF ITS PROFILE DISTRIBUTION IN CONTAMINATED PODZOLIC SOIL
}

\author{
Rachkova N.G., Shaposhnikova L.M.
}

Institute of Biology of FRC Komi Scientific Centre of Ural Branch of RAS, Syktyvkar, e-mail:nata.rachkova67@mail.ru

For modeling of behavior of pollutants in taiga ecosystems, long-term distribution and transformation of radium-226 speciation in upper 50-centimeter layer of typical podzolic soil were studied, the statistical relationships between parameters of these processes and soil physicochemical characteristics were investigated. It was revealed that upon long-term contact, watersoluble compounds of radionuclide are transformed and firmly bound in soil absorbing complex. At same time, main stock of mobile radium is deposited in soil layer $(0-20) \mathrm{cm}$ in composition of potentially mobile compounds, which are extracted with $1 \mathrm{~mol} / \mathrm{dm}^{3}$ of hydrochloric acid, which is important for selection of methods for decontamination of soils that are similar in physical and chemical composition and soil formation type. In soil layer deeper than $20 \mathrm{~cm}$, no more than $11,2 \%$ of radium amount in thickness $(0-50) \mathrm{cm}$ migrates. In total, about $95 \%$ of radium stock is concentrated in acid-soluble and fixed fractions, readily mobile watersoluble and exchangeable radionuclide forms provide 1 and $4 \%$ of radium amount in soil layer $(0-50) \mathrm{cm}$. The results of linear regression and correlation analyzes indicate, that under radioactive contamination, radium differentiation in profile of podzolic soils is controlled by distribution of calcium compounds. To approximate indicators of radium migration, mathematical models are proposed, the quality of which is estimated by determination coefficients $0,467-0,997$. The results of modeling have shown that interaction of iron and calcium compounds determines radionuclide mobility in contaminated soil. The data obtained can be used to predict the distribution and localization of radium in ecosystems.

Keywords: radioactive contamination, podzolic soil, radium-226, activity concentration, speciation of radionuclide, distribution in soil profile, regression analysis

Актуальность исследования связана с задачами прогнозирования миграции и дезактивации грунтов, загрязненных долгоживущими естественными радионуклидами. Такие из них, как изотопы радия, являются высокорадиоактивными химическими аналогами щелочноземельных элементов, типоморфных для почв и жизненно важных для организмов. Геохимические аспекты прогнозирования поведения радия по-прежнему мало разработаны [1-3].

Цель исследования - моделирование поведения радия-226 в загрязненных эко- системах среднетаёжной подзоны (Республика Коми) на основе изучения статистической связи между параметрами вертикальной миграции и трансформации форм нахождения радионуклида в типичной подзолистой почве и ее физико-химическими характеристиками.

\section{Материалы и методы исследования}

Эксперимент проводили в среднетаежной подзоне Республики Коми на равнинном участке с типичной подзолистой почвой, образованной на крупнопылеватых 
покровных суглинках. Глубина ее промерзания не превышает 40 см. Почва под толщей (25-50 см) слабо водопроницаема. Под подстилкой находится подзолистый горизонт с множеством конкреций. По их граням наблюдаются гумусово-глинистые пленки и осветленный пылеватый материал.

Для проведения эксперимента участок разделили на 12 делянок (по 6 делянок для опытов А и Б). В соответствии с вариантами опыта слой почвы 0-20 см на делянках загрязнили двумя разными количествами раствора хлорида радия. За счет этого его содержания в почве слоя 0-20 см существенно превысили соответствующее фоновое содержание радионуклида.

Спустя 14 лет из толщи 0-50 см загрязненной почвы отобрали пробы на определение валового содержания и форм нахождения радионуклида, оценивали почвенные физико-химические характеристики [4, 5]. Для исследования форм нахождения радий-226 последовательно десорбировали из почвы дистиллированной водой (водорастворимая), 1 моль/дм ${ }^{3} \mathrm{CH}_{3} \mathrm{COONH}_{4}$ (обменная) и 1 моль/дм ${ }^{3} \mathrm{HCl}$ (кислоторастворимая) при времени контакта почвы с каждым экстрагентом 1 сутки, комнатной температуре, соотношении фаз 1:5. Недесорбированную часть радионуклида считали фиксированной в почве. Радий-226 в образцах определяли эманационным методом с ошибкой измерений менее $15 \%$ [6]. Стандартными методами [7] оценивали долю калия, натрия, кремния, титана в почве (в расчете на их оксиды), гидролитическую кислотность, $\mathrm{pH}$ водный и солевой, содержания гумуса, поглощенных катионов магния и кальция, валовое содержание фосфатов и калия. Химические анализы выполнены в лабораториях миграции радионуклидов и радиохимии и «Экоаналит» Института биологии ФИЦ Коми НЦ УрО РАН. Данные обработаны с использованием программы Statistica.

\section{Результаты исследования и их обсуждение}

В незагрязненной почве экспериментального участка содержание радия-226 было понижено по сравнению со средними значениями для Восточно-Европейской равнины. Между формами нахождения с разной подвижностью радионуклид был распределен равномерно. При валовом содержании $16,4 \pm 0,9$ мБк/Г его удельная активность в водорастворимой, обменной и кислоторастворимой фрак- циях составляла от 3,9 до 4,4 мБк/г. Водородный показатель почвы варьировался от 5,02 до 5,45, содержание поглощенных катионов магния - от 0,38 до 1,74 , кальция - от 0,56 до 2,87 мг-экв/100 г. Содержание кальция, магния, железа и алюминия (в расчете на оксиды) изменялось в градиентах 0,18-0,27, 2,97-5,86, 1,44$1,87,7,88-10,07 \%$.

Сразу после загрязнения концентрации радия-226 в 0-20 см почвы делянок превышали его фоновое содержание примерно в 80 и 100 раз (в вариантах эксперимента А и Б соответственно). За 14 лет после загрязнения из этого количества в нижележащую толщу вытеснилось за счет почвенных процессов около $11 \%$ радионуклида (табл. 1). При этом распределение радия-226 в профиле 0-50 см характеризовалось преимущественным депонированием в слое почвы 0-20 см наряду с достоверным повышением валового содержания и, одновременно, снижением содержания подвижных форм нахождения радионуклида в слое 25-30 см. Удельная активность водо- и кислоторастворимой фракций радия в этих образцах была достоверно ниже, чем для почвы горизонта 20-25 см.

В толще почвы 0-20 см с максимальной концентрацией радия сосредоточено в среднем 89 и $94 \%$ его количества в вариантах опыта А и Б (табл. 2). При этом доля мобильных форм нахождения (сумма водорастворимой, обменной и кислоторастворимой форм) здесь составила 48 и $75 \%$ от валового содержания радионуклида соответственно (рис. 1). Из них на долю кислоторастворимой фракции приходилось подавляющее количество радия в слое (свыше 46 и 71\% удельной активности). На глубине 20-25 см в обоих вариантах опыта валовое содержание элемента достоверно снижалось $\mathrm{p}<0,001$ и 0,01$)$, значительно уменьшались удельные активности его мобильных форм и относительные содержания слабосвязанного в почве радионуклида, экстрагированного дистиллированной водой и раствором ацетата аммония. На глубине 25-30 см отмечалось повышение в 2 раза валового содержания элемента по сравнению со слоями 2025 и 30-35 см. При этом подвижность соединений радия в почве снижалась до минимума, в том числе за счет уменьшения количества радионуклида в составе водорастворимых и кислоторастворимых фракций. В варианте опыта Б с более высоким уровнем загрязнения почвы спад содержа- 
ния первых составил 15 раз, вторых - около 100 раз. В слое 25-30 см радионуклид был депонирован преимущественно за счет его фиксации в почве. На этой глубине заметно доминирование фракции подвижных соединений радия, экстрагируемых рас- твором ацетата аммония (вариант Б). Эта специфика свидетельствует об изменении механизмов поглощения радионуклида, а значительная вариабельность параллельных определений содержания радия в слое указывает на обратимость этих процессов.

Таблица 1

Удельная активность радия-226 и его мобильных форм нахождения в толще $0-50$ см загрязненной почвы

\begin{tabular}{|c|c|c|c|c|c|}
\hline Вариант экс- $\begin{array}{c}\text { Почвенный } \\
\text { перимента }\end{array}$ & Валовая удельная & \multicolumn{2}{|c|}{ Удельная активность мобильных форм, мБк/г } \\
\cline { 3 - 6 } слой, см & активность, мБк/г & водорастворимых & обменных & кислоторастворимых \\
\hline \multirow{4}{*}{ А } & $0-20$ & $884 \pm 80$ & $7 \pm 4$ & $17 \pm 3$ & $400 \pm 180$ \\
\cline { 2 - 6 } & $20-25$ & $38 \pm 9$ & $2,0 \pm 0,4$ & $9 \pm 4$ & $20 \pm 3$ \\
\cline { 2 - 6 } & $25-30$ & $81 \pm 16$ & $1,5 \pm 0,4$ & $13 \pm 5$ & $8 \pm 5$ \\
\cline { 2 - 6 } & $30-35$ & $13 \pm 4$ & $16 \pm 6$ & $12 \pm 4$ & $4 \pm 1$ \\
\cline { 2 - 6 } & $35-40$ & $12 \pm 3$ & $1 \pm 1$ & $12 \pm 9$ & $6 \pm 1$ \\
\cline { 2 - 6 } & $40-50$ & $14 \pm 3$ & $0,7 \pm 0,5$ & $11 \pm 6$ & $4 \pm 2$ \\
\hline \multirow{6}{*}{ Б } & $0-20$ & $590 \pm 30$ & $3,3 \pm 0,7$ & $16 \pm 7$ & $420 \pm 50$ \\
\cline { 2 - 6 } & $20-25$ & $52 \pm 12$ & $1,5 \pm 0,6$ & $3 \pm 1$ & $16 \pm 7$ \\
\cline { 2 - 6 } & $25-30$ & $100 \pm 70$ & $0,2 \pm 0,1$ & $13 \pm 6$ & $4,8 \pm 0,4$ \\
\cline { 2 - 6 } & $30-35$ & $22 \pm 5$ & $1,1 \pm 0,7$ & $8 \pm 3$ & $6 \pm 2$ \\
\cline { 2 - 6 } & $35-40$ & $18 \pm 5$ & не обнаружено & $3 \pm 1$ & $5 \pm 3$ \\
\cline { 2 - 6 } & $40-50$ & $31 \pm 8$ & $1,5 \pm 0,7$ & $4 \pm 1$ & $9 \pm 1$ \\
\hline
\end{tabular}

Таблица 2

Запасы радия-226 в загрязненной почве (верхняя строка - вариант опыта А, нижняя - Б)

\begin{tabular}{|c|c|c|c|c|c|c|}
\hline Почвенный слой, см & $0-20$ & $20-25$ & $25-30$ & $30-35$ & $35-40$ & $40-50$ \\
\hline Запасы радия, & 407,0 & 45,0 & 103,4 & 26,2 & 28,4 & 47,4 \\
кБк* & 271,0 & 61,1 & 131,1 & 30,1 & 28,2 & 93,3 \\
\hline Содержание радия, \% & 94,2 & 1,0 & 2,4 & 0,6 & 0,7 & 1,1 \\
запаса, в том числе & 88,8 & 2,0 & 4,3 & 1,0 & 0,9 & 3,1 \\
водорастворимых & 0,7 & 0,06 & 0,04 & 0,3 & 0,04 & 0,05 \\
& 0,5 & 0,06 & 0,01 & 0,05 & 0,00 & 0,25 \\
обменных & 1,8 & 0,2 & 0,4 & 0,2 & 0,4 & 0,7 \\
кислоторастворимых & 2,4 & 0,1 & 0,5 & 0,4 & 0,2 & 0,4 \\
& 43,4 & 0,3 & 0,2 & 0,1 & 0,2 & 0,3 \\
\hline
\end{tabular}
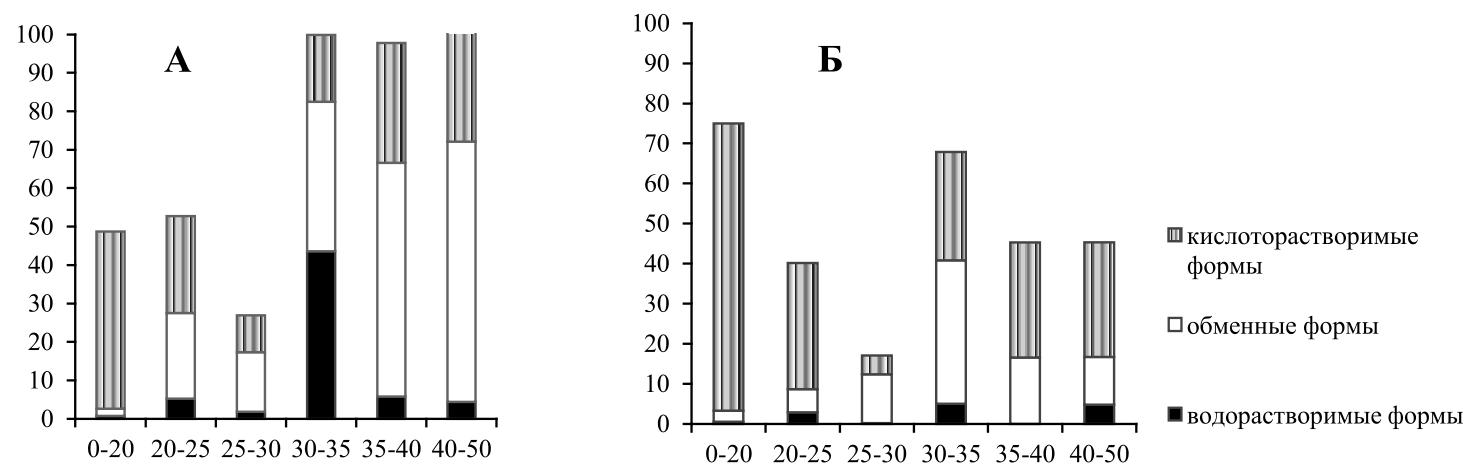

Рис. 1. Доля удельной активности мобильных форм нахождения радия-226 (в\% его валовой удельной активности в слое) в толще 0-50 см загрязненной почвы 
Увеличение количества обменного радионуклида на глубине 25-30 см и фиксация радия в слое могут обусловливаться его сорбцией по механизму ионного обмена на коллоидных соединениях неорганической и органической природы и на глинистых минералах, а также соосаждением с гуматами кальция или окклюзией радионуклида в структурах коллоидных частиц. Усиление прочности поглощения радия в слое 25-30 см возможно вследствие оптимального соотношения в нем концентраций органических веществ и обменных форм нахождения кальция [4, 5]. В работах [2, 8-10] также отмечается влияние этих почвенных показателей на депонирование радия в фоновых и загрязненных почвах.

В нижних слоях подзолистого горизонта наряду с возрастанием содержания радионуклида отмечается увеличение его запасов, экстрагирующихся в обменную и кислоторастворимую фракции, накоплению которых способствует уплотнение почвы. В целом в ходе эксперимента установлено, что при многолетнем контакте водорастворимые соединения радия, поступившие в подзолистую почву, трансформируются и прочно связываются в почвенном поглощающем комплексе. В толще 0-50 см основной запас мобильных форм радиоактивного элемента депонировался в составе кислоторастворимых соединений, потенциально подвижных в почве. В то же время подвижные водорастворимые и обменные формы радионуклида обеспечивали 1 и $4 \%$ его количества в толще $0-50$ см.

Таким образом, из всех почвенных образцов радий наиболее эффективно экстрагировался 1 моль/дм ${ }^{3} \mathrm{HCl}$. При подсчете запасов радионуклида в вышеуказанной фракции обнаруживалось свыше 44 и 69\% от его активности в почве в вариантах опыта (рис. 2). Это важно для выбора оптимальных подходов к дезактивации почв, подобных исследуемым грунтам по физико-химическому составу и типу почвообразования. Суммарно в кислоторастворимой и фиксированной фракциях сосредоточено $95 \%$ запаса радия.

Статистическая обработка полученных данных в связи с физико-химическими характеристиками почвы свидетельствовала, что по коэффициенту корреляции Пирсона удельная активность радионуклида, находящегося в почве в водорастворимой форме, тесно связана с концентрацией обменного кальция и железа $(0,70)$, что рассматривается нами как результат сорбции радия железо-гумусовыми комплексными соединениями кальция $[4,5]$. Удельная активность радиоэлемента, депонированного в почве в обменной форме нахождения, также слабо коррелировала с процентными содержаниями алюминия $(0,32)$ и гумуса $(0,27)$ в почвенной массе, с содержанием железа $(-0,28)$, гигроскопической влаги $(0,34)$ и почвенной кислотностью (около -0,3) $[4,5]$. В то же время кислоторастворимые соединения радия были дифференцированы в толще почвы в соответствии со слабой линейной зависимостью от распределения железа $(0,29)$, магния $(-0,36)$, кремния $(0,35)$ и гигроскопической влаги $(-0,31)[4,5]$, что может быть маркером поглощения радионуклида окристаллизованными и коллоидными соединениями железа. Возможна конкуренция этих механизмов поглощения и обменной сорбции радионуклида органо-минеральными коллоидами гидроксидов алюминия. Примечательно, что содержания обменных и кислоторастворимых форм нахождения радионуклида статистически связаны с одними и теми же характеристиками почвы [4, 5]. Установленные парные линейные корреляции часто слабы и противоположны по знаку, что подтверждает конкурентность процессов, лежащих в их основе.
A

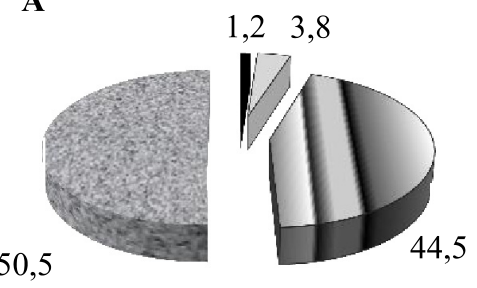

Б

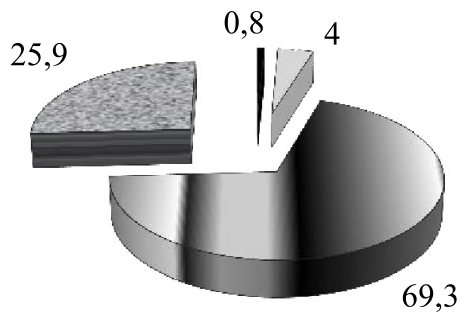

ш водорастворимые формы

口обменные формы

س кислоторастворимые формы

■фиксированные формы

Рис. 2. Распределение запаса мобильных и фиксированных форм радия-226 в почве 
Таблица 3

Статистическая связь распределения радия-226 в толще почвы 0-50 см и её характеристик

\begin{tabular}{|c|c|c|c|c|c|c|c|}
\hline \multirow{2}{*}{$\begin{array}{c}\text { Зависимая } \\
\text { переменная }\end{array}$} & \multirow[t]{2}{*}{ Модель } & \multirow{2}{*}{$\begin{array}{l}\text { Независимые } \\
\text { переменные }\end{array}$} & \multirow{2}{*}{$\begin{array}{c}\text { Коэффициенты } \\
\text { регрессии }\end{array}$} & \multicolumn{4}{|c|}{ Характеристики модели } \\
\hline & & & & $\mathrm{R}^{2}$ & $\mathrm{~S}_{\mathrm{R}}$ & $\mathrm{F}$ & $P_{f}$ \\
\hline \multirow{2}{*}{$\begin{array}{l}\mathrm{C}_{\mathrm{Ra}}, \mathrm{MБ \kappa} / \Gamma \\
\mathrm{C}_{\mathrm{Ra}}, \mathrm{MБ \kappa} / \Gamma\end{array}$} & 1 & $\mathrm{C}_{\mathrm{Ca}}, \mathrm{M \Gamma -ЭКВ/100} \mathrm{г}$ & 451,2 & 0,997 & 17,2 & 687,8 & 0,000 \\
\hline & 2 & $\begin{array}{l}\text { a) } \mathrm{C}_{\mathrm{Ca}},{ }^{\mathrm{M \Gamma}-э К в} / 100 \text { г } \\
\text { б) } \mathrm{C}_{\mathrm{CaO}}, \%\end{array}$ & $\begin{array}{l}423,3 \\
782,5\end{array}$ & 0,990 & $\begin{array}{c}13,7 \\
185,9\end{array}$ & 712,0 & $\begin{array}{l}0,000 \\
0,001\end{array}$ \\
\hline \multirow{2}{*}{$\mathrm{C}_{\text {Rа вод }}, \mathrm{MБК/ \Gamma}$} & 1 & $\mathrm{C}_{\mathrm{Ca}}, \mathrm{MГ-ЭКВ/100} \mathrm{Г}$ & 1,93 & 0,467 & 0,516 & 14,0 & 0,002 \\
\hline & 2 & $\begin{array}{l}\text { a) } \mathrm{C}_{\mathrm{Ca}},{ }_{1} \text { Мг-эКв } / 100 \Gamma \\
\text { б) } \mathrm{C}_{\mathrm{Fe} 2 \mathrm{O} 3}, \%\end{array}$ & $\begin{array}{l}2,60 \\
6,79\end{array}$ & 0,697 & $\begin{array}{l}0,448 \\
0,014\end{array}$ & 17,2 & $\begin{array}{l}0,000 \\
0,004\end{array}$ \\
\hline $\mathrm{C}_{\mathrm{Ra} \mathrm{кис}}, \mathrm{MБК/Г}$ & 1 & $\mathrm{C}_{\mathrm{Ca}}, \mathrm{M \Gamma -ЭКВ/100} \mathrm{Г}$ & 329,8 & 0,908 & 26,2 & 157,9 & 0,000 \\
\hline
\end{tabular}

П р и м е ч а н и я : $\mathrm{R}^{2}$ - коэффициент детерминации и $\mathrm{S}_{\mathrm{R}}$ - его стандартное отклонение; $\mathrm{F}$ - критическое значение критерия Фишера и $\mathrm{P}_{\mathrm{f}}-$ его уровень значимости. Для включения независимой переменной $\mathrm{F} \geq 3,840$. $\mathrm{C}_{\mathrm{Ra}}, \mathrm{C}_{\mathrm{Ra}}$ вод, $\mathrm{C}_{\mathrm{Ra} \text { кис }}$ - валовая удельная активность радия-226, его водорастворимых и кислоторастворимых форм нахождения. $C_{C a}$ - содержание в почве поглощенного катиона кальция, $C_{\mathrm{CaO}}, C_{\mathrm{Fe}_{2} \mathrm{O}_{3}}$ - валовые содержания (в расчете на оксиды) кальция и железа (III) соответственно.

Для моделирования поведения радия в исследуемой почве был использован пошаговый регрессионный анализ. Суждение о формах нахождения радионуклидов в почве, основываясь исключительно на уравнениях регрессии, сопряжено с большой неопределенностью, однако их анализ в совокупности с результатами последовательной экстракции выявляет значимость свойств загрязненной почвы в трансформации форм нахождения радионуклидов. Результаты моделирования миграции радия-226 с использованием регрессионного анализа (табл. 3) свидетельствуют, что его профильная дифференциация в подзолистых почвах зоны тайги полностью контролируется процессами вертикального распределения соединений кальция.

За счет этих процессов прочность сорбции радионуклида в почвенных слоях увеличивается.

Так, распределение валового радия при подзолистом типе почвообразования на 99, 7 \% зависит от поведения поглощенного катиона кальция, который как предиктор представлен во всех линейных регрессионных моделях миграции радия в почве. Кроме того, этот показатель используется для описания распределения кислоторастворимых форм радия, составляющих основную долю его почвенных соединений. Удельная активность водорастворимых форм радиоэлемента аппроксимируется уравнением с двумя слагаемыми, один из которых пропорционален содержанию поглощенных катионов кальция $[4,5]$, а другой - доле соединений железа в почвенной массе (в расчете на оксиды).

\section{Заключение}

В результате проведенного модельного эксперимента выявлены закономерности распределения радия-226 в широко распространенной в таежной зоне подзолистой почве в условиях её загрязнения радийсодержащими растворами. Выявлено, что при многолетнем контакте водорастворимые соединения радионуклида трансформируются и прочно связываются в почвенном поглощающем комплексе. Распределение радия-226 в профиле почвы 0-50 см характеризуется преимущественным депонированием в слое 0-20 см наряду с достоверным повышением валового содержания и, одновременно, снижением содержания подвижных форм нахождения радионуклида в слое 25-30 см. Основной запас мобильных форм нахождения радия депонирован в слое 0-20 см в составе потенциально подвижных соединений, экстрагирующихся 1 моль/дм ${ }^{3}$ соляной кислотой, что важно для выбора методов дезактивации грунтов, подобных исследованному по физико-химическому составу и типу почвообразования. Суммарно в кислоторастворимой и фиксированной фракциях сосредоточено около $95 \%$ запаса радия, легкоподвижные водорастворимые и обменные формы радионуклида обеспечивают $5 \%$ его количества в толще почвы 0-50 см. Выраженное изменение подвижности радия в почвенных слоях может обуславливаться концентрациями в них обменного кальция, соединений железа и органических веществ. Это подтверждается не только данными литературы, но и результатами линейного 
регрессионного и корреляционного анализов, которые свидетельствуют, что в условиях радиоактивного загрязнения профильная дифференциация радия в почвах этого типа контролируется процессами распределения соединений кальция. Качество аппроксимации показателей миграции радия в предлагаемых моделях оценено коэффициентами детерминации в диапазоне значений 0,467-0,997. Выявлено, что возможность миграции радия в загрязненной почве определяется взаимодействием соединений железа и кальция. Предложенные модели миграции радионуклида могут быть использованы при прогнозировании его рассеяния и локализации в сходных по типу почвы экосистемах. Практическая значимость работы связана с разработкой мер дезактивации территорий Республики Коми, загрязненных вследствие добычи радия из минерализованных подземных вод.

Исследования выполнены в рамках Госзадания № АAAA-A18-118011190102-7 при частичной поддержке проекта РФФИ и Правительства Республики Коми № 2045-110009p_a.

\section{Список литературы / References}

1. Giffroy P., Durrieu G., Garnier J.-M. Probabilistic distribution coefficients $\left(\mathrm{K}_{\mathrm{d}} \mathrm{s}\right)$ in freshwater for radioisotopes of $\mathrm{Ag}$, $\mathrm{Am}, \mathrm{Ba}, \mathrm{Be}, \mathrm{Ce}, \mathrm{Co}, \mathrm{Cs}$, I, Mn, Pu, Ra, Ru, Sb, Sr and Thimplications for uncertainty analisys of models simulating the transport of radionuclides in rivers. J. Environm. Radioactivity. 2009. Vol. 100. P. 785-794. DOI: 10.1016/j.jenvrad.2008.10.

2. Abdullah J.A., Al-Masri M.S., Amin Y., Awad I., Sheaib Z. Chemical fractionation of radium-226 in NORM contaminated soil from oilfields. J. Environm. Radioactivity. 2016. Vol. 165. P. 47-53. DOI: 10.1016/j.jenvrad.2016.09.003.

3. Guillen J., Munoz-Serrano A., Salvador Baeza A., Salas A. Speciation of naturally occurring radionuclides in Mediter- ranean soils: bioavailabilty assessment. Environm. Sci. Poll. Res. 2018. Vol. 25. P. 6772-6782. DOI: 10.1007/s11356-017-1021-z.

4. Рачкова Н.Г., Зайнуллин В.Г. Моделирование подвижности радия-226 в загрязнённых подзолистых почвах на основе данных регрессионного анализа // Актуальные проблемы региональной экологии и биодиагностика живых систем: материалы XIII Всероссийской научно-практической конференции с международным участием (г. Киров, 1-2 декабря 2015 г.). В 2-х книгах. Книга 2. Киров: Издво ООО «Веси», 2015. С. 194-197.

Rachkova N.G., Zaynullin V.G. Modeling of the mobility of radium-226 in contaminated podzolic soils based on regression analysis data // Aktualniye problemy regionalnoy ecologii i biodiagnostika zhivyh sistem: materialy' Vserossijskoy nauchno-prakticheskoy konferenzii s mezhdunarodnym uchastiem (g. Kirov, 1-2 dekabrya 2015 g.). V 2h knigah. Kniga 2. Kirov: Izd-vo OOO «Vesi», 2015. P. 194-197 (in Russian).

5. Рачкова Н.Г. Роль сорбентов в процессах трансформации соединений урана, радия и тория в подзолистой почве: автореф. дис. ... канд. биол. наук. Сыктывкар, 2006. $22 \mathrm{c}$.

Rachkova N.G. The role of sorbents in the transformation of uranium, radium and thorium compounds in podzolic soil: avtoref. dis. ... kand. biol. nauk. Syktyvkar, 2006. 22 p. (in Russian).

6. Rachkova N.G., Shaposhnikova L.M. Speciation of radium-226 in the components of terrestrial and aqueous northern taiga ecosystems in a former radium production site. Geochemistry International. 2020. Vol. 58. No. 6. P. 719-728. DOI: $10.31857 / \mathrm{S} 0016752520050106$

7. Аринушкина Е.В. Руководство по химическому анализу почв. М.: Изд-во МГУ, 1962. 491 с.

Arinushkina E.V. Manual for Chemical Analysis of Soils. M.: Izd-vo MGU, 1962. 491 p. (in Russian).

8. Noskova L.M., Shuktomova I.I. Radium distribution in anthropogenic soils as a function of soil physicochemical and mineralogical parameters.Geochemistry International. 2015. Vol. 53. No. 11. P. 1012-1018. DOI: 10.1134/S0016702915090050.

9. Beznosikov V.A., Lodygin E.D., Shuktomova I.I. Artificial and natural radionuclides in soils of the southern and middle taiga zones of Komi republic. Eurasian Soil Science. 2017. Vol. 50. No. 7. P. 814-819. DOI: 10.1134/S1064229317050039.

10. Pearson A.J., Gam S., Hermanspahn N., Glover C.N., Anderson C.W.N. Radium in New Zealand agricultural soils: Phosphate fertiliser inputs, soil activity concentrations and fractionation profiles. J. Environm. Radioactivity. 2019. Vol. 205206. P. 119-126. DOI: 10.1016/j.jenvrad.2019.05.010. 